\title{
ON THE WELL-POSEDNESS OF THE KIRCHHOFF STRING
}

\author{
ALBERTO AROSIO AND STEFANO PANIZZI
}

\begin{abstract}
Let us consider the Cauchy problem for the quasilinear hyperbolic integro-differential equation

$$
\begin{array}{ll}
u_{t t}-m\left(\int_{\Omega}\left|\nabla_{x} u\right|^{2} d x\right) \triangle_{x} u=f(x, t) & (x \in \Omega, t>0), \\
u(\cdot, t)_{\mid \partial \Omega}=0 & (t \geq 0),
\end{array}
$$

where $\Omega$ is an open subset of $\mathbb{R}^{n}$ and $m$ is a positive function of one real variable which is continuously differentiable. We prove the well-posedness in the Hadamard sense (existence, uniqueness and continuous dependence of the local solution upon the initial data) in Sobolev spaces of low order.
\end{abstract}

\section{INTRODUCTION}

Let $m(\cdot):[0,+\infty[\longrightarrow] 0,+\infty[$ be any continuos function and let $\Omega$ be any open subset of $\mathbb{R}^{n}$. We are concerned with the following integro-differential equation, which as in $[\mathrm{AG}]$ we call Kirchhoff equation:

$$
\begin{array}{ll}
u_{t t}-m\left(\int_{\Omega}\left|\nabla_{x} u\right|^{2} d x\right) \triangle_{x} u=f(x, t) & (x \in \Omega, t>0), \\
u(\cdot, t)_{\mid \partial \Omega}=0 & (t \geq 0) .
\end{array}
$$

Eq. (1) for $n=1$ and

$$
m(r)=c_{0}^{2}+r
$$

was introduced in 1876 by KIRCHHOFF [Ki] as a nonlinear model of the free transversal vibrations of a clamped string. G. F. CARRIER [Ca] and R. NARASHIMA [Na] recovered Eq.(1), without quoting Kirchhoff. See also [Ka] [NM] [Am]. D. W. OPLINGER [Op] compared numerical and experimental results. We refer the reader to the survey [Ar] for references about Eq.(1) from a physical point of view.

From the pure mathematical point of view, Eq.(1) may be considered as the simplest example of quasilinear evolution equation of hyperbolic type.

There are therefore two good reasons to study Eq.(1): for its intrinsic physical meaning, and as a prototype for more complicated equations. A variety of papers have been produced on the existence and uniqueness of the solution of the Cauchy

Received by the editors April 25, 1994 and, in revised form, January 30, 1995.

1991 Mathematics Subject Classification. Primary 35L70, 35B30; Secondary 34G20.

Key words and phrases. Well-posedness, quasilinear hyperbolic equation, extensible string, local existence, Ritz-Galerkin approximation.

The research was supported by the $40 \%$ funds of the Italian Ministero della Università e della Ricerca Scientifica e Tecnologica. 
problem for Eq.(1): we refer the reader to the survey [Ar] for a (possibly incomplete) commented list. For reasons of space, we do not consider here any generalization of Eq.(1) and we limit ourselves to list known papers about Eq.(1) in three classes: 1) local existence in Sobolev spaces: [Be] [Di1] [Me] [Ri] [MM] [AG] [Ma];

$\left.1^{\prime}\right)$ same as above, but degenerate $(m \geq 0)$ : $[\mathrm{Ni}$ ] [Eb] [EMM] [Ya] [Yam] [Ga] [AG]; 2 ) special cases of global existence in Sobolev spaces. Special $m(\cdot)$ : [Po2]. Travelling wave solutions in $\Omega:=\mathbb{R}^{n}:$ [Di2]. $\Omega:=\mathbb{R}^{n}$ and small data: [GH] [DS2]. See also $[\mathrm{BL}]$.

3) global existence for analytic initial data: $[\mathrm{Be}][\mathrm{Po} 1][\mathrm{Ni}][\mathrm{Li}][\mathrm{AS}][\mathrm{Nis}][\mathrm{DS} 1][\mathrm{Ar}]$.

The outstanding question for Eq.(1) is the global solvability for $C^{\infty}$ initial data. In this paper we treat another question which seems to be not yet explicitly considered: the well-posedness in Hadamard's classical sense of the Cauchy problem for Eq.(1). Actually, a straightforward application of T. KATO's general theory [K2] for abstract evolution equations would permit us to decide the question affirmatively. However we felt the exigency of examining the question in its details, and of providing accurate estimates of the solution, for the purpose of investigating the continuity modulus of the

resolvent map: (initial data) $\longmapsto$ (solution).

We first prove accurate a priori estimates for the energy of a solution, which yield local well-posedness in Hadamard's sense for the Cauchy problem for Eq.(1) (Theorems 4.1-4.2). As a byproduct, Ritz-Galerkin approximants converge uniformly in the phase space on a suitable time interval to a function, which is the unique solution (Corollaries 4.1-4.2). Such a proof of existence and uniqueness differs substantially from KATO's point of view, which adopts Banach's contraction principle. This result improves all the existence/uniqueness results of the group 1) above. For instance: in [AG] the open set $\Omega$ was assumed to satisfy Poincaré's inequality and the technique of proof was based on a fixed point argument, so no mention was made of Ritz-Galerkin approximations; Matos in [Ma] removed the coerciveness assumption on the operator $-\triangle$, allowing $\Omega$ to be any open subset of $\mathbb{R}^{n}$, but uniqueness was established only for higher Sobolev exponent and the convergence of the Ritz-Galerkin sequence was proven to hold true only in a weak sense. Also, our method provides an accurate estimate of the energy, which should permit us, for instance, to treat in a future paper also the mildly degenerate case $m \geq 0, m\left(\int_{\Omega}\left|\nabla x u_{0}\right|^{2} d x\right)>0$, in the spirit of [Ga].

We discover that the modulus of continuity of the map (2) depends only upon the fixed initial data (and may actually behave very badly), and we describe subsets of initial data with uniform modulus. In particular we investigate conditions which guarantee the Hölder continuity of the map (2).

We note that the two crucial points of our proof of the well-posedness are: (i) a splitting of the difference of two different solutions (this device appears already in [K3]); (ii) the use of the spectral decomposition of the operator $-\triangle$.

Because of the presence of the integro-differential term, we have found that the most profitable way to study Eq.(1) is to reformulate it as an evolution equation in a Hilbert space. 
The plan of the paper is as follows:

Section 2. Statement of the main result (the well-posedness of the Cauchy-Dirichlet problem for Eq.(1)).

Section 3. Preliminaries: abstract framework.

Section 4. A priori estimates.

Section 5 and Section 6. Proof of the main result.

Section 7. Study of the continuity modulus of the resolvent map (2), which in general is not Hölder continuous.

Appendix.

\section{Statement of the Main Result: Well-Posedness of the CAUChY-Dirichlet PROBlem For EQ.(1)}

Since we want to study Eq.(1) in Sobolev spaces of fractional order, we recall the concrete ${ }^{1}$ characterization of the domains

$$
V_{\alpha}(\Omega):=D\left(A^{\alpha / 2}\right)
$$

of the fractional powers of the operator

$$
\begin{aligned}
& D(A):=H^{2}(\Omega) \bigcap H_{0}^{1}(\Omega), \\
& A:=-\triangle_{x}:=\sum_{i=1}^{n} \frac{\partial^{2}}{\partial x_{i}^{2}},
\end{aligned}
$$

where $\Omega$ is either (i) any open bounded subset of $\mathbb{R}^{n}$ with boundary $\partial \Omega$ of $C^{\infty}$ class, or (ii) the half-space $\mathbb{R}_{+}^{n}:=\left\{\left(x_{1}, \ldots, x_{n}\right) \in \mathbb{R}^{n}: x_{n} \geq 0\right\}$, or (iii) $\Omega \equiv \mathbb{R}^{n}$.

In the cases (i), (ii) the characterization is due to D. FUJIWARA [Fu] (cf. [LM]). Let us recall it.

For $\alpha \geq 0, \quad \alpha=2 k+\alpha^{\prime}, k \in \mathbb{N}, 0 \leq \alpha^{\prime}<2$, the space $V_{\alpha}$ as follows is defined: $V_{\alpha}(\Omega)=\left\{u \in H^{\alpha}(\Omega):\left(-\triangle_{x}\right)^{j} u \in H_{0}^{1}(\Omega)\right.$ for $\left.0 \leq j \leq k-1, \quad\left(-\triangle_{x}\right)^{k} u \in B^{\alpha^{\prime}}\right\}$,

where

$$
\begin{gathered}
B^{\alpha^{\prime}}(\Omega):=H^{\alpha^{\prime}}(\Omega) \text { for } 0 \leq \alpha^{\prime}<1 / 2, \\
B^{\alpha^{\prime}}(\Omega):=\left\{u \in H^{\alpha^{\prime}}(\Omega): u_{\mid \partial \Omega}=0\right\} \text { for } 1 / 2<\alpha^{\prime}<2, \alpha^{\prime} \neq 3 / 2 .
\end{gathered}
$$

In the exceptional cases $\alpha^{\prime}=1 / 2, \alpha^{\prime}=3 / 2$ we have:

$$
\begin{gathered}
B^{1 / 2}(\Omega):=\left\{u \in H^{1 / 2}(\Omega): \int_{\Omega} d^{-1}(x)|u(x)|^{2} d x<\infty\right\}, \\
B^{3 / 2}(\Omega):=\left\{u \in H^{3 / 2}(\Omega) \cap H_{0}^{1}(\Omega): \int_{\Omega} d^{-1}(x)\left|\tilde{D}_{j} u(x)\right|^{2} d x<\infty, 0 \leq j \leq n-1\right\},
\end{gathered}
$$

1 For any bounded $\Omega$, the space $V_{\alpha}(\Omega)$ may be characterized in the following abstract way. Let $\left\{v_{k}\right\}$ be an orthonormal basis such that for each $k=1,2, \ldots$

$$
A v_{k}=\lambda_{k}^{2} v_{k}
$$

we have that $u \in V_{\alpha}(\Omega)$ if and only if

$$
\sum_{k=1}^{+\infty} k^{2 \alpha / n}\left|\int_{\Omega} u(x) \overline{v_{k}(x)} d x\right|^{2}<+\infty
$$


where $d(x):=\operatorname{dist}(x, \partial \Omega)$, and $\left(\tilde{D}_{j}\right)_{0 \leq j \leq n-1}$ is a system of first order differential operators, the restriction of which to $\partial \Omega$ forms a basis of the tangent space to $\partial \Omega$.

Finally, in case (iii) we simply have

$$
V_{\alpha}\left(\mathbb{R}^{n}\right):=H^{\alpha}\left(\mathbb{R}^{n}\right) .
$$

In the following we denote by $|\cdot|$ the norm in $L^{2}(\Omega)$ and by $|\cdot|_{V_{\alpha}}$ the seminorm $\left|A^{\alpha / 2}(\cdot)\right|$. As for the norm in the space $V_{\alpha}(\Omega)$, we set

$$
\|\cdot\|_{V_{\alpha}}:=\left(c|\cdot|^{2}+|\cdot|_{V_{\alpha}}^{2}\right)^{1 / 2}
$$

where $c$ is any positive number in the cases (ii), (iii) and $c=0$ in the case (i).

We need the following definition

Definition 2.1. We say that the function $m$ is coercive if and only if there exists a constant $\nu$ such that

$$
m(s) \geq \nu>0 \quad(s \geq 0) .
$$

We say that the function $m$ is coercive at $\infty$ if and only if

$$
m(s)>0 \quad(s \geq 0)
$$

and

$$
\int_{0}^{+\infty} m(s) d s=+\infty
$$

Let us set

$$
M(r):=\int_{0}^{r} m(s) d s \quad(r \geq 0) .
$$

Given a pair $\left(u_{0}, u_{1}\right)$ in $H^{1}(\Omega) \times L^{2}(\Omega)$ let us define the Hamiltonian [Be]

$$
\mathcal{H}\left(u_{0}, u_{1}\right):=M\left(\int_{\Omega}\left|\nabla_{x} u_{0}\right|^{2} d x\right)+\int_{\Omega}\left|u_{1}(x)\right|^{2} d x
$$

and the quantities $\nu\left(u_{0}, u_{1}\right)$ and $L\left(u_{0}, u_{1}\right)$ as the best constants such that

$$
\begin{gathered}
M(r) \leq \mathcal{H}\left(u_{0}, u_{1}\right) \quad \Longrightarrow \quad m(r) \geq \nu\left(u_{0}, u_{1}\right), \\
{\left[M(r) \leq \mathcal{H}\left(u_{0}, u_{1}\right), M(s) \leq \mathcal{H}\left(u_{0}, u_{1}\right)\right]} \\
\Longrightarrow|m(r)-m(s)| \leq L\left(u_{0}, u_{1}\right)|r-s| .
\end{gathered}
$$

Note that if $m$ is a locally Lipschitz function coercive at $\infty$, we have (see Proposition 6.2 below)

$$
\nu\left(u_{0}, u_{1}\right)>0, \quad L\left(u_{0}, u_{1}\right)<+\infty .
$$

We remark that part (a) of the following theorem, $i$. e. existence and uniqueness in Sobolev spaces of order $\geq 3 / 2$, was already proven, by using a fixed point technique, in $[\mathrm{AG}]$ under the assumption: $\Omega$ contained in a bounded strip of $\mathbb{R}^{n}$, namely $-\triangle$ strictly positive. Moreover in $[\mathrm{Ma}]$ the existence of a solution in Sobolev spaces of order $\geq 3 / 2$ was proven without restrictions on the set $\Omega$, but the uniqueness was proven only for solutions in Sobolev spaces of order $\geq 2$. The technique of proof in [Ma] was based on the spectral decomposition of the operator $-\triangle$, in order to construct a sequence of Ritz-Galerkin approximations converging in a weak star topology to a solution. 
We also note that the life span exhibited in $[\mathrm{AG}]$ is improved by a factor 4 .

Theorem 2.1 (main result). Let $\Omega$ be either any bounded open subset of $\mathbb{R}^{n}$ with $C^{\infty}$ boundary or the half-space $\mathbb{R}_{+}^{n}$ or $\Omega \equiv \mathbb{R}^{n}$. Let $m$ be any locally Lipschitz function coercive at $\infty$, and let $\alpha \geq 3 / 2$.

Then (a) for any pair of initial data $\left(u_{0}, u_{1}\right) \in V_{\alpha}(\Omega) \times V_{\alpha-1}(\Omega)$ and any function $f \in L_{l o c}^{1}\left(\left[0,+\infty\left[; V_{\alpha-1}(\Omega)\right)\right.\right.$, there exists a unique solution

$$
u \in C^{0}\left(\left[0, T^{* *}\left[; V_{\alpha}(\Omega)\right) \cap C^{1}\left(\left[0, T^{* *}\left[; V_{\alpha-1}(\Omega)\right),\right.\right.\right.\right.
$$

of the problem

$$
\begin{cases}u_{t t}-m\left(\int_{\Omega}\left|\nabla_{x} u\right|^{2} d x\right) \triangle_{x} u=f(x, t) & (x \in \Omega, t>0), \\ u(x, 0)=u_{0}(x), \quad u_{t}(x, 0)=u_{1}(x) & (x \in \Omega), \\ u(\cdot, t) \in V_{\alpha}(\Omega), \quad u_{t}(\cdot, t) \in V_{\alpha-1}(\Omega) & (t \geq 0) .\end{cases}
$$

Let us introduce the time $T^{*}$

$$
T^{*}:=\frac{\nu\left(u_{0}, u_{1}\right)^{3 / 2}}{L\left(u_{0}, u_{1}\right)\left(m\left(\int_{\Omega}\left|\nabla x u_{0}\right|^{2} d x\right)\left|u_{0}\right|_{V_{3 / 2}(\Omega)}^{2}+\left|u_{1}\right|_{V_{1 / 2}(\Omega)}^{2}\right)} .
$$

The critical time $T^{* *}$ is implicitly defined by the formula

$$
\begin{aligned}
T^{*}=T^{* *}\left\{1+\left(m\left(\int_{\Omega}\left|\nabla_{x} u_{0}\right|^{2} d x\right)\left|u_{0}\right|_{V_{3 / 2}(\Omega)}^{2}\right.\right. & \left.+\left|u_{1}\right|_{V_{1 / 2}(\Omega)}^{2}\right)^{-1 / 2} \\
& \left.\times \int_{0}^{T^{* *}}|f(\cdot, s)|_{V_{1 / 2}(\Omega)} d s\right\} .
\end{aligned}
$$

Moreover: (b) the solution is the uniform limit of the whole sequence of its Ritz-Galerkin approximations; (c) the solution depends continuously upon the data; namely, for every $T<T^{*}$, the map

$$
\begin{aligned}
\text { data } & \longmapsto \text { solution } \\
V_{\alpha} \times V_{\alpha-1} \times L^{1}\left([0, T] ; V_{\alpha-1}(\Omega)\right) & \longrightarrow C^{0}\left([0, T] ; V_{\alpha}\right) \cap C^{1}\left([0, T] ; V_{\alpha-1}\right)
\end{aligned}
$$

is continuous at the point $\left(u_{0}, u_{1}, f\right)$.

The above statement follows by a straightforward application of the abstract results given in Section 4 (note: in the cases (ii), (iii) one works with an arbitrary $c>0$, and then lets $c \rightarrow 0^{+}$).

\section{Preliminaries: abstract Framework}

To reformulate Eq. (1) as an evolution equation in a Hilbert space, we need some preliminaries and notations.

Let $V$ and $H$ be real or complex Hilbert spaces, normed respectively by $\|\cdot\|$ and $|\cdot|, V \subseteq H$. If $V^{\prime}$ denotes the (anti)dual of $V$, we have then $V \subseteq H \subseteq V^{\prime}$, in the sense that the duality bracket $\langle\cdot, \cdot\rangle:=\langle\cdot, \cdot\rangle_{V \times V^{\prime}}$ coincides with the inner product $(\cdot, \cdot):=(\cdot, \cdot)_{H}$ on $H \times V$.

Let $A: V \longrightarrow V^{\prime}$ be a linear bounded operator, symmetric in the sense that

$$
\langle A v, w\rangle=\overline{\langle A w, v\rangle} \quad(w \in V, v \in V) .
$$


Moreover assume that for some $c(A) \geq 0$ and $\eta(A)>0$,

$$
\begin{array}{ll}
\langle A v, v\rangle \geq 0 & (v \in V), \\
\langle A v, v\rangle+c(A)|v|^{2} \geq \eta(A)\|v\|^{2} & (v \in V) .
\end{array}
$$

The operator $(A+c(A))$ turns out to be an isomorphism of $V$ onto $V^{\prime}$. We denote $D:=D(A):=\{v \in V: A v \in H\}$. It is $D\left(A+c I_{H}\right)=D$ for every $c \in \mathbb{R}$. The operator

$$
A_{c}:=\left(A+c I_{H}\right): D \longrightarrow H \quad(c \geq c(A)),
$$

turns out to be a self-adjoint positive definite operator in $H$, hence $A: D \longrightarrow H$ is a self-adjoint nonnegative operator in $H$.

In this paper we establish results for the Cauchy problem for the abstract evolution equation, $(\cdot)^{\prime}:=\frac{d}{d t}$,

$$
u^{\prime \prime}+m(\langle A u, u\rangle) A u=f \quad(t>0),
$$

but for the sake of clearness in calculations, we will give complete proofs only in the case when $f \equiv 0$, i.e. for the equation

$$
u^{\prime \prime}+m(\langle A u, u\rangle) A u=0 \quad(t>0) .
$$

with initial data

$$
u(0)=u_{0}, \quad u^{\prime}(0)=u_{1} .
$$

If we consider any open set $\Omega \subseteq \mathbb{R}^{n}$ and we set

$$
V:=H_{0}^{1}(\Omega), \quad H:=L^{2}(\Omega) \quad \text { and } \quad A:=-\triangle_{x}:=\sum_{i=1}^{n} \frac{\partial^{2}}{\partial x_{i}^{2}},
$$

then Eq. (9) reads as Eq. (1). Moreover we may choose

$$
c(A)=\left\{\begin{array}{l}
0 \text { in the case (i), } \\
\text { any } c>0 \text { in the cases (ii), (iii). }
\end{array}\right.
$$

Hereafter we set for simplicity's sake

$$
c:=c(A) .
$$

We will base our spectral analysis upon the operator $A^{1 / 2}$, which in the concrete case of the Kirchhoff string (1) is a first order (pseudodifferential) operator.

For every $\alpha \in \mathbb{R}$, we will consider the power $A_{c}^{\alpha / 2}$ (namely the $(\alpha / 2)^{t h}$ power of $\left.A_{c}\right)$, see e.g. [Ru]. For $\alpha \geq 0$, the space $V_{\alpha}:=D\left(A_{c}^{\alpha / 2}\right)=D\left(A^{\alpha / 2}\right)$ is made a Hilbert space under the norm

$$
\|u\|_{\alpha}:=\left|A_{c}^{\alpha / 2} u\right| .
$$

We note that $V_{\alpha} \subseteq V_{\beta}$ for $\beta \leq \alpha$, with continuous and dense injection. For $\alpha<0, V_{\alpha}$ is defined as the (anti)dual space of $V_{-\alpha}$, endowed with the (anti)dual norm, which will be still denoted by $\|\cdot\|_{\alpha}$. In any case $V_{\alpha}$ and $H$ turn out to be isomorphic via $\left(A_{c}^{\alpha / 2}\right)^{t}$, where $(\cdot)^{t}$ denotes transposition (for $\alpha \geq 0$ this means that $\left(A_{c}^{\alpha / 2}\right)^{t}$ extends $A_{c}^{\alpha / 2}$, i.e. $\left.\left(A_{c}^{\alpha / 2}\right)_{\mid V_{\alpha}}^{t}=A_{c}^{\alpha / 2}\right)$. More generally we have

$$
\begin{array}{ll}
\left(A_{c}^{\beta / 2}\right)_{\mid V_{\alpha}}^{t}=\left(A_{c}^{(\beta-\alpha) / 2}\right)^{t} A_{c}^{\alpha / 2} & \text { for } 0 \leq \alpha \leq \beta, \\
\left(A_{c}^{\beta / 2}\right)_{\mid V_{\alpha}}^{t}=A_{c}^{(\beta-\alpha) / 2}\left(A_{c}^{\alpha / 2}\right)^{t} & \text { for } \beta \leq \alpha \leq 0,
\end{array}
$$

so that $V_{\alpha}$ and $V_{\alpha-\beta}$ are isomorphic via $\left(A_{c}^{\beta / 2}\right)^{t}$ for each $\alpha \in \mathbb{R}, \beta \in \mathbb{R}$. 
The phase space for Eq.(10) will be

$$
\mathcal{E}_{\alpha}:=V_{\alpha} \times V_{\alpha-1} \quad(\alpha \in \mathbb{R}),
$$

and we set, for any continuous nonnegative function $a(\cdot)$ and $u \in C^{0}\left([0, T] ; \mathcal{E}_{\alpha}\right),{ }^{2}$

$$
\begin{aligned}
E_{\alpha, a}(t, u) & :=a(t)\left\|A^{1 / 2} u(t)\right\|_{\alpha-1}^{2}+\left\|u^{\prime}(t)\right\|_{\alpha-1}^{2} \\
& =a(t)\left|A_{c}^{(\alpha-1) / 2} A^{1 / 2} u(t)\right|^{2}+\left|A_{c}^{(\alpha-1) / 2} u^{\prime}(t)\right|^{2} \quad(t \geq 0) .
\end{aligned}
$$

\section{A PRIORI ESTIMATES}

From now on, we set for brevity

$$
m_{(u)}(t):=m(\langle A u(t), u(t)\rangle)
$$

Let $f$ be any function in $L_{l o c}^{1}\left(\left[0,+\infty\left[; V_{1 / 2}\right)\right.\right.$ and let $\left(u_{0}, u_{1}\right) \in \mathcal{E}_{3 / 2}$. In the following statement we will need the notation $(t \geq 0)$

$$
\eta\left(t ; f, u_{0}, u_{1}\right):=\left\{1+\left(m\left(\left\langle A u_{0}, u_{0}\right\rangle\right)\left\|A^{1 / 2} u_{0}\right\|_{1 / 2}^{2}+\left\|u_{1}\right\|_{1 / 2}^{2}\right)^{-1 / 2} \int_{0}^{t}\|f(s)\|_{1 / 2} d s\right\}^{2} .
$$

Theorem 4.1 (a priori estimates). Let us assume that the operator $A$ satisfies condition (8), and that there exist two numbers $\nu$ and $L$ such that

$$
\begin{cases}m(r) \geq \nu>0 & (r \geq 0) \\ |m(r)-m(s)| \leq L|r-s| & (r \geq 0, s \geq 0) .\end{cases}
$$

Let $u \in C^{0}\left([0, T] ; \mathcal{E}_{\alpha_{0}}\right), \alpha_{0} \geq 3 / 2$, be a solution of $(9)-(11)$, i.e.

$$
\left\{\begin{array}{l}
u^{\prime \prime}+m_{(u)}(t) A u=f(t) \quad(t>0) \\
u(0)=u_{0}, \quad u^{\prime}(0)=u_{1}
\end{array}\right.
$$

and let $w \in C^{0}\left([0, T] ; \mathcal{E}_{\beta}\right), \beta \in \mathbb{R}$, be a solution of

$$
w^{\prime \prime}+m_{(u)}(t) A w=g(t) \quad(t>0), \quad \text { with } g \in L^{1}\left(0, T ; V_{\beta-1}\right) .
$$

Let us introduce the quantity $\left.\left.T^{*}:=T^{*}\left(u_{0}, u_{1}\right) \in\right] 0,+\infty\right]$ as ${ }^{3}$

$$
T^{*}:=T^{*}\left(u_{0}, u_{1}\right):=\frac{\nu^{3 / 2}}{L E_{3 / 2, m_{(u)}}(0, u)},
$$

and let $T^{* *}:=T^{* *}\left(u_{0}, u_{1}, f\right)$ be the quantity implicitly defined as

$$
T^{*}=T^{* *} \eta\left(T^{* *} ; f, u_{0}, u_{1}\right) .
$$

Then, for every $\alpha \leq \alpha_{0}$, the following a priori estimates hold true for $0 \leq t<$ $T^{* *}:$

$$
E_{\alpha, m_{(u)}}(t, u) \leq \frac{\left(\left(E_{\alpha, m_{(u)}}(0, u)\right)^{1 / 2}+\int_{0}^{t}\|f(s)\|_{\alpha-1} d s\right)^{2}}{1-\eta\left(t ; f, u_{0}, u_{1}\right) t / T^{*}}
$$

\footnotetext{
2 This is a short notation to mean that $\left(u, u^{\prime}\right) \in C^{0}\left([0, T] ; \mathcal{E}_{\alpha}\right)$.

3 When $T^{*}=+\infty$ any fraction with $T^{*}$ in the denominator is meant to vanish.
} 


$$
\begin{aligned}
\left(E_{\beta, m_{(u)}}(t, w)\right)^{1 / 2} \leq & \left(E_{\beta, m_{(u)}}(0, w)\right)^{1 / 2} \\
& +\int_{0}^{t}\left(\frac{\left(E_{\beta, m_{(u)}}(s, w)\right)^{1 / 2}}{2\left(T^{*} \eta\left(s ; f, u_{0}, u_{1}\right)^{-1}-s\right)}+\|g(s)\|_{\beta-1}\right) d s,
\end{aligned}
$$

and

$$
E_{\beta, m_{(u)}}(t, w) \leq \frac{\left(\left(E_{\beta, m_{(u)}}(0, w)\right)^{1 / 2}+\int_{0}^{t}\|g(s)\|_{\beta-1} d s\right)^{2}}{1-\eta\left(t ; f, u_{0}, u_{1}\right) t / T^{*}} .
$$

Proof. In order that the schema of proof appear clear, we give the proof only for the case $f \equiv 0$. Let us set

$$
\begin{aligned}
& y_{\beta}(t):=\left(E_{\beta, m_{(u)}}(t, w)\right)^{1 / 2}, \\
& \varphi(t):=\frac{L}{2 \nu^{3 / 2}} E_{3 / 2, m_{(u)}}(t, u) .
\end{aligned}
$$

Let us assume for the moment that $w$ belongs to $C^{0}\left([0, T] ; \mathcal{E}_{\beta+1}\right)$ : then by Eq.(13) we get that for a. a. $t \in[0, T]$

$$
\begin{aligned}
\frac{1}{2} \frac{d}{d t}\left(y_{\beta}^{2}\right) & =m^{\prime}(\langle A u, u\rangle) \operatorname{Re}\left(A u, u^{\prime}\right)\left|A_{c}^{(\beta-1) / 2} A^{1 / 2} u\right|^{2}+\operatorname{Re}\left(A_{c}^{(\beta-1) / 2} g, A_{c}^{(\beta-1) / 2} u^{\prime}\right) \\
& \leq \varphi y_{\beta}^{2}+\|g(s)\|_{\beta-1} y_{\beta} .
\end{aligned}
$$

By dividing by $y_{\beta}$ we get

$$
\frac{d}{d t}\left(y_{\beta}\right) \leq \varphi y_{\beta}+\|g(s)\|_{\beta-1}
$$

By integrating we get for $0 \leq t \leq T$

$$
y_{\beta}(t) \leq y_{\beta}(0)+\int_{0}^{t}\left(\varphi(s) y_{\beta}+\|g(s)\|_{\beta-1}\right) d s .
$$

Now, due to the fact that Eq.(13) is linear in $w$, estimate (18) holds true also for any function $w$ which is simply in $C^{0}\left([0, T] ; \mathcal{E}_{\beta}\right)$. We can therefore apply (18) for the choice $w=u, g \equiv 0$ and any $\alpha \leq \alpha_{0}$ in place of $\beta$, to get

$$
\left(E_{\alpha, m_{(u)}}(t, u)\right)^{1 / 2} \leq\left(E_{\alpha, m_{(u)}}(0, u)\right)^{1 / 2}+\int_{0}^{t} \varphi(s)\left(E_{\alpha, m_{(u)}}(s, u)\right)^{1 / 2} d s .
$$

In particular for $\alpha=3 / 2$ we get, by a standard comparison principle for ordinary differential equations and by (14), that

$$
\varphi(t) \leq \frac{1}{\frac{1}{\varphi(0)}-2 t}=\frac{1}{2\left(T^{*}-t\right)} \quad\left(0 \leq t<T^{*} \wedge T\right),
$$

hence by (19), via the Gronwall lemma, that (15) holds true for any $\alpha \leq \alpha_{0}$.

Condition (16) follows immediately by (18) and (20); (17) follows in turn from the Gronwall lemma. Q.E.D. 
Remark 4.1. Estimate (15) is the best estimate that we are able to provide for Eq.(10), however we are not able to prove that it is sharp. As a matter of fact no counterexample to the global existence for the Cauchy problem for Eq.(10) has been exhibited up to now .

\section{Proof of the Main RESUlt}

Now we state, in abstract form, our basic result. In order to make the exposition easier, in this section we will make some simplifying assumptions, namely:

1) $m$ is globally (not merely locally) Lipschitz continuous, and it is bounded;

2) the source term $f$ vanishes identically.

We will discuss the general case in the next section.

For any subset $W \subseteq \mathcal{E}_{3 / 2}$ and any function $m$ satisfying (12), let us introduce the following quantities:

$$
\begin{gathered}
E:=E(W):=\sup _{\left(u_{0}, u_{1}\right) \in W} m\left(\left\langle A u_{0}, u_{0}\right\rangle\right)\left\|A^{1 / 2} u_{0}\right\|_{1 / 2}^{2}+\left\|u_{1}\right\|_{1 / 2}^{2}, \\
T^{*}:=T^{*}(W):=\frac{\nu^{3 / 2}}{L E} \in[0,+\infty] .
\end{gathered}
$$

If $W$ is bounded in $\mathcal{E}_{3 / 2}$ it is not difficult to prove that (see Proposition 6.2 in the next section)

$$
E<\infty \quad \text { and } \quad 0<T^{*} \leq \infty .
$$

Theorem 5.1 (continuous dependence upon the data). Let us assume that the operator A satisfies condition (8), and that

(i) $m$ satisfies (12) and

$$
m(r) \leq \Lambda \quad(r \geq 0)
$$

(ii) $W$ is a bounded set of $\mathcal{E}_{3 / 2}$.

Let the quantities $E$ and $T^{*}$ be defined as in (21), (22). Let $\alpha_{0}$ and $T$ be real numbers such that

$$
\begin{gathered}
\alpha_{0} \geq 3 / 2, \\
0<T<T^{*} .
\end{gathered}
$$

Consider any given solution $u \in C^{0}\left([0, T] ; \mathcal{E}_{\alpha_{0}}\right)$ to the problem (10)-(11) with initial data in $W$. Let finally $\alpha$ be given such that

$$
1 / 2 \leq \alpha \leq \alpha_{0}
$$

Then, for every $\varepsilon>0$, there exists a number $\delta$ which depends only upon the quantities $\varepsilon, \nu, \Lambda, u_{0}, u_{1}, \alpha, T / T^{*}$, such that

$$
E_{\alpha, \nu}(0, v-u)<\delta \Longrightarrow E_{\alpha, \nu}(t, v-u)<\varepsilon \quad(0 \leq t \leq T),
$$

for every $v$ in $C^{0}\left([0, T] ; \mathcal{E}_{\alpha_{0}}\right)$ which solves problem (10)-(11) with initial data in $W$.

Special case: for $\alpha \leq 1 / 2$ we have a Lipschitz dependence upon the initial data, i.e. the number $\delta$ depends in a linear way upon the quantity $\varepsilon$ (with coefficient depending only upon the quantities $\left.\Lambda / \nu, T / T^{*}\right)$. 
Proof. We prove the statement under the following simplifying assumption:

$$
\alpha_{0}=3 / 2
$$

Let $u$ and $v$ be as in the statement, and let us denote by $\left(u_{0}, u_{1}\right)$ and respectively $\left(v_{0}, v_{1}\right)$ their initial data. Following [K3], in order to estimate the $\alpha^{t h}$-energy of the difference $v-u$, we split it in two parts. For this we need an auxiliary function $z$ in $C^{0}\left([0, T] ; \mathcal{E}_{\alpha}\right)$, defined as the solution to the linear "hybrid" problem:

$$
\left\{\begin{array}{l}
z^{\prime \prime}+m_{(v)} A z=0 \quad(0<t<T), \\
z(0)=u_{0} \\
z^{\prime}(0)=u_{1}
\end{array}\right.
$$

Then we set

$$
w_{1}:=v-z \quad \text { and } \quad w_{2}:=z-u,
$$

so that

$$
w_{1}+w_{2}=v-u \text {. }
$$

The first term $w_{1}$ is an "innocuous" one. Indeed it satisfies the following linear problem with vanishing source term

$$
\left\{\begin{array}{l}
w_{1}^{\prime \prime}+m_{(v)} A w_{1}=0 \quad(0<t<T) \\
w_{1}(0)=v_{0}-u_{0} \\
w_{1}^{\prime}(0)=v_{1}-u_{1}
\end{array}\right.
$$

so that we may apply Theorem 4.1 (namely estimate (17)) to get for any $\beta \leq 3 / 2$

$$
E_{\beta, m_{(v)}}\left(t, w_{1}\right) \leq \frac{E_{\beta, m_{(v)}}(0, v-u)}{1-t / T^{*}} \quad(0 \leq t \leq T) .
$$

On the other hand, $w_{2}$ solves the problem

$$
\left\{\begin{array}{l}
w_{2}^{\prime \prime}+m_{(v)} A w_{2}=\left(m_{(u)}-m_{(v)}\right) A u \quad(0<t<T), \\
w_{2}(0)=0 \\
w_{2}^{\prime}(0)=0 .
\end{array}\right.
$$

To simplify the notation, we set, for any $\beta \leq 3 / 2$,

$$
y_{\beta}(t):=\left(E_{\beta, m_{(v)}}\left(t, w_{2}\right)\right)^{1 / 2} .
$$

We can apply Theorem 4.1 (estimate (16)) for any

$$
\beta \leq 1 / 2
$$

to get

$$
y_{\beta}(t) \leq \int_{0}^{t}\left(\frac{y_{\beta}(s)}{2\left(T^{*}-s\right)}+\left|\left(m_{(u)}-m_{(v)}\right)(s)\right| \cdot\|A u(s)\|_{\beta-1}\right) d s .
$$


By arguing as in $[\mathrm{AG}]$ and by using condition (12), the split (27), estimates (15) and (28) for $\beta=1 / 2$, we have for $0 \leq t \leq T$

$$
\begin{aligned}
\left|\left(m_{(u)}-m_{(v)}\right)(t)\right| \leq & \left.L|| A^{1 / 2} v\right|^{2}-\left|A^{1 / 2} u\right|^{2} \mid \\
= & L\left|\left(A_{c}^{1 / 4} A^{1 / 2}(u+v), A_{c}^{-1 / 4} A^{1 / 2}(v-u)\right)\right| \\
\leq & \frac{L}{\nu^{1 / 2}}\left(\left(E_{3 / 2, m_{(v)}}(t, v)\right)^{1 / 2}+\left(E_{3 / 2, m_{(u)}}(t, u)\right)^{1 / 2}\right) \\
& \quad \times\left(\left\|A^{1 / 2} w_{1}\right\|_{-1 / 2}+\left\|A^{1 / 2} w_{2}\right\|_{-1 / 2}\right) \\
& \leq \frac{2 L E^{1 / 2}}{\nu\left(1-t / T^{*}\right)^{1 / 2}}\left(\frac{\left(E_{1 / 2, m_{(v)}}(0, v-u)\right)^{1 / 2}}{\left(1-t / T^{*}\right)^{1 / 2}}+y_{1 / 2}(t)\right) .
\end{aligned}
$$

Moreover, since

$$
\|A \cdot\|_{\beta-1} \leq\left\|A^{1 / 2} \cdot\right\|_{\beta},
$$

thanks to (15) we have

$$
\|A u(t)\|_{\beta-1} \leq \frac{\left(E_{\beta+1, m_{(u)}}(0, u)\right)^{1 / 2}}{\nu^{1 / 2}\left(1-t / T^{*}\right)^{1 / 2}} \quad(0 \leq t \leq T) .
$$

By putting (32) and (33) into (31) we obtain

$$
\begin{aligned}
& y_{\beta}(t) \leq \frac{1}{2} \int_{0}^{t} \frac{y_{\beta}(s) d s}{\left(T^{*}-s\right)} \\
& +\frac{2 L E^{1 / 2}}{\nu^{3 / 2}}\left(E_{\beta+1, m_{(u)}}(0, u)\right)^{1 / 2} \int_{0}^{t}\left(\frac{\left(E_{1 / 2, m_{(v)}}(0, v-u)\right)^{1 / 2}}{\left(1-s / T^{*}\right)^{3 / 2}}+\frac{y_{1 / 2}(s)}{1-s / T^{*}}\right) d s .
\end{aligned}
$$

Thanks to (24) and (30) we can choose $\beta:=1 / 2$ in (34). From the definitions of $E$ and $T^{*}$ we then get

$$
y_{1 / 2}(t) \leq \frac{5}{2} \int_{0}^{t} \frac{y_{1 / 2}(s) d s}{\left(T^{*}-s\right)}+2\left(T^{*} E_{1 / 2, m_{(v)}}(0, v-u)\right)^{1 / 2} \int_{0}^{t} \frac{d s}{\left(T^{*}-s\right)^{3 / 2}} .
$$

Now by a standard comparison technique we obtain for $0 \leq t \leq T$,

$$
y_{1 / 2}(t) \leq\left(E_{1 / 2, m_{(v)}}(0, v-u)\right)^{1 / 2} \frac{\left(2-t / T^{*}\right)}{\left(1-t / T^{*}\right)^{5 / 2}} t / T^{*} .
$$

If we re-insert the estimate (35) into (34) and we set now $\beta=\alpha$, thanks to the trivial equality

$$
1+\frac{(2-x) x}{(1-x)^{2}}=\frac{1}{(1-x)^{2}}
$$

we get the estimate for $0 \leq t \leq T$,

$$
\begin{aligned}
y_{\alpha}(t) \leq & \frac{1}{2} \int_{0}^{t} \frac{y_{\alpha}(s) d s}{\left(T^{*}-s\right)} \\
& +\frac{2 L E^{1 / 2}}{\nu^{3 / 2}}\left(E_{\alpha+1, m_{(u)}}(0, u) E_{1 / 2, m_{(v)}}(0, v-u)\right)^{1 / 2} \int_{0}^{t} \frac{d s}{\left(1-s / T^{*}\right)^{7 / 2}}
\end{aligned}
$$


If $T^{*}=\infty$, i.e. by (22) $L E=0$, the above inequality gives immediately $y_{\alpha} \equiv 0$. In the case $T^{*}<\infty$, by a standard comparison result and (22) we get the following generalization of (35):

$$
y_{\alpha}(t) \leq\left(\frac{E_{\alpha+1, m_{(u)}}(0, u) E_{1 / 2, m_{(v)}}(0, v-u)}{E}\right)^{1 / 2} \cdot \frac{\left(2-t / T^{*}\right)}{\left(1-t / T^{*}\right)^{5 / 2}} t / T^{*} .
$$

By using (27), (28) for $\beta=\alpha$, and (37) we get that (in the case when $v$ and $u$ have the same initial data the right-hand side of the following inequality is meant to vanish)

$\left(E_{\alpha, \nu}(t, v-u)\right)^{1 / 2} \leq\left(E_{\alpha, m_{(v)}}(t, v-u)\right)^{1 / 2}$

$$
\begin{aligned}
& \leq \frac{\left(E_{\alpha, m_{(v)}}(0, v-u)\right)^{1 / 2}}{\left(1-t / T^{*}\right)^{5 / 2}} \\
& \times \max \left\{1,\left(\frac{E_{\alpha+1, m_{(u)}}(0, u)}{E}\right)^{1 / 2} \cdot\left(\frac{E_{1 / 2, m_{(v)}}(0, v-u)}{E_{\alpha, m_{(v)}}(0, v-u)}\right)^{1 / 2}\right\} .
\end{aligned}
$$

If $\alpha=1 / 2$, then by (23) we get

$$
E_{1 / 2, \nu}(t, v-u) \leq \frac{\Lambda}{\nu} \frac{E_{1 / 2, \nu}(0, v-u)}{\left(1-t / T^{*}\right)^{5}} .
$$

This provides our assertion for the case $\alpha=1 / 2$. Unfortunately, (38) does not (apparently) permit us to prove it for any other value of $\alpha$.

The difficulty is related to the fact that $w_{2}$ satisfies no equation with vanishing source term. However, we have found that by combining the estimates (35) and (28) with the spectral decomposition of $w_{2}$ we can overcome this difficulty. The idea is that the approximation of $w_{2}$ (up to a fixed frequency $n$ ) may be controlled in a Lipschitz fashion, with Lipschitz constant growing as the accuracy of the approximation increases. On the other hand, the rest of $w_{2}$ becomes smaller and smaller as the frequency $n$ increases. Then, one concludes by a standard $\varepsilon-\delta$ argument.

Let $E(\lambda)$ denote the spectral measure associated to the self-adjoint nonnegative operator $A^{1 / 2}$ (see e.g. $[\mathrm{Ru}]$ ) and $\chi_{G}$ the characteristic function of a subset $G$ of the real line. For each $n \in \mathbb{N}$, let us define the following orthogonal projections in $H$ :

$$
P_{n}:=\int \chi_{[0, n]}(\lambda) d E(\lambda), \quad Q_{n}:=\int \chi_{] n,+\infty[}(\lambda) d E(\lambda) .
$$

Since $P_{n}+Q_{n}=I_{H}$, we may split

$$
w_{2}=P_{n} w_{2}+Q_{n} w_{2} .
$$

We may split the energy

$$
E_{\alpha, \nu}\left(t, w_{2}\right)=E_{\alpha, \nu}\left(t, P_{n} w_{2}\right)+E_{\alpha, \nu}\left(t, Q_{n} w_{2}\right) .
$$


Moreover, for $\alpha \geq 1 / 2$, thanks to (12), (29), (35), (23) and (36) we have

$$
\begin{aligned}
E_{\alpha, \nu}\left(t, P_{n} w_{2}\right) & \leq\left(n^{2}+c\right)^{\alpha-1 / 2} E_{1 / 2, \nu}\left(t, P_{n} w_{2}\right) \\
& \leq\left(n^{2}+c\right)^{\alpha-1 / 2} E_{1 / 2, \nu}\left(t, w_{2}\right) \\
& \leq\left(n^{2}+c\right)^{\alpha-1 / 2} y_{1 / 2}^{2}(t) \\
& \leq\left(n^{2}+c\right)^{\alpha-1 / 2} \frac{\Lambda}{\nu} \frac{E_{1 / 2, \nu}(0, v-u)}{\left(1-t / T^{*}\right)^{5}}
\end{aligned}
$$

On the other hand, we note that $Q_{n} u$ and $Q_{n} z$ solve the following problems with vanishing source term:

$$
\left\{\begin{array} { l } 
{ ( Q _ { n } u ) ^ { \prime \prime } + m _ { ( u ) } A Q _ { n } u = 0 , } \\
{ Q _ { n } u ( 0 ) = Q _ { n } u _ { 0 } , } \\
{ Q _ { n } u ^ { \prime } ( 0 ) = Q _ { n } u _ { 1 } , }
\end{array} \quad \text { and } \quad \left\{\begin{array}{l}
\left(Q_{n} z\right)^{\prime \prime}+m_{(v)} A Q_{n} z=0, \\
Q_{n} z(0)=Q_{n} u_{0}, \\
Q_{n} z^{\prime}(0)=Q_{n} u_{1} .
\end{array}\right.\right.
$$

Let us define for each $n \in \mathbb{N}$

$$
h_{n, \alpha}\left(u_{0}, u_{1}\right):=\nu\left|Q_{n} A_{c}^{(\alpha-1) / 2} A^{1 / 2} u_{0}\right|^{2}+\left|Q_{n} A_{c}^{(\alpha-1) / 2} u_{1}\right|^{2} .
$$

Thanks to (12) and estimate (17) of Theorem 4.1 and (23), we get

$$
E_{\alpha, \nu}\left(t, Q_{n} u\right) \leq E_{\alpha, m_{(u)}}\left(t, Q_{n} u\right) \leq \frac{E_{\alpha, m_{(u)}}\left(0, Q_{n} u\right)}{1-t / T^{*}} \leq \frac{\Lambda}{\nu} \frac{h_{n, \alpha}\left(u_{0}, u_{1}\right)}{1-t / T^{*}} .
$$

The analogous estimate holds true for $Q_{n} z$. Summing up, $Q_{n} w_{2}=Q_{n} z-Q_{n} u$ satisfies

$$
E_{\alpha, \nu}\left(t, Q_{n} w_{2}\right) \leq \frac{4 \Lambda}{\nu} \frac{h_{n, \alpha}\left(u_{0}, u_{1}\right)}{1-t / T^{*}} .
$$

We note that the relevant feature of (43) is that the right hand side does not depend upon $\left(v_{0}, v_{1}\right)$, which can vary in all of $W$, but only upon $\left(u_{0}, u_{1}\right)$, which is fixed.

Therefore, by (40), (41) and (43) we get for $0 \leq t \leq T$

$$
E_{\alpha, \nu}\left(t, w_{2}\right) \leq\left(n^{2}+c\right)^{\alpha-1 / 2} \frac{\Lambda}{\nu} \frac{E_{1 / 2, \nu}(0, v-u)}{\left(1-t / T^{*}\right)^{5}}+\frac{4 \Lambda}{\nu} \frac{h_{n, \alpha}\left(u_{0}, u_{1}\right)}{1-t / T^{*}},
$$

and finally from $(27),(28)$ for $\beta=\alpha$ and $(23) \quad(0 \leq t \leq T)$

$$
\begin{aligned}
E_{\alpha, \nu}(t, v-u) \leq \frac{2 \Lambda}{\nu\left(1-T / T^{*}\right)}\left\{\left(n^{2}+c\right)^{\alpha-1 / 2} \frac{E_{1 / 2, \nu}(0, v-u)}{\left(1-T / T^{*}\right)^{4}}\right. & \\
& \left.+E_{\alpha, \nu}(0, v-u)+4 h_{n, \alpha}\left(u_{0}, u_{1}\right)\right\} .
\end{aligned}
$$

Now fix a number $\varepsilon>0$. Since $\left(u_{0}, u_{1}\right)$ belongs to $\mathcal{E}_{3 / 2}$ and (26) holds true, there exists a natural number $\bar{n}$ such that

$$
\frac{8 \Lambda}{\nu\left(1-T / T^{*}\right)} h_{\bar{n}, \alpha}\left(u_{0}, u_{1}\right)<\frac{\varepsilon}{3} \text {. }
$$

Moreover we have still by (26)

$$
E_{1 / 2, \nu}(0, v-u) \leq K^{1 / 2-\alpha} E_{\alpha, \nu}(0, v-u), \quad \text { where } K:=\frac{\|i\|_{V, H}^{2}}{\eta(A)} .
$$


Then any number $\delta>0$ such that

$$
\delta \frac{2 \Lambda}{\nu\left(1-T / T^{*}\right)}\left(1+\left(\frac{\bar{n}^{2}+c}{K}\right)^{\alpha-1 / 2} \cdot \frac{1}{\left(1-T / T^{*}\right)^{4}}\right)<\frac{2}{3} \varepsilon
$$

satisfies the thesis. Q.E.D.

In Theorem 5.1 we have employed the energy

$$
E_{\alpha, \nu}(t, u):=\nu\left\|A^{1 / 2} u(t)\right\|_{\alpha-1}^{2}+\left\|u^{\prime}(t)\right\|_{\alpha-1}^{2}
$$

since this quantity provides neat estimates. Unfortunately, it has a drawback: when $c>0$, that is, in the concrete case when $\Omega$ does not satisfy the Poincaré inequality, $E_{\alpha, \nu}$ may be not positive definite. However, we can slightly modify $E_{\alpha, \nu}$ to have a positive definite quantity which fits Theorem 5.1 as well as $E_{\alpha, \nu}$. Let us introduce the following modified energy for a function $u \in C^{0}\left([0, T] ; \mathcal{E}_{\alpha}\right)$ :

$$
\tilde{E}_{\alpha, \nu}(t, u):=\nu\|u(t)\|_{\alpha}^{2}+\left\|u^{\prime}(t)\right\|_{\alpha-1}^{2}
$$

Theorem 5.2. The statement of Theorem 5.1 holds true too with $\tilde{E}_{\alpha, \nu}(t, u)$ in place of $E_{\alpha, \nu}(t, u)$.

Proof. For every $w \in C^{0}\left([0, T] ; \mathcal{E}_{\alpha}\right)$, we have

$$
\tilde{E}_{\alpha, \nu}(t, w)=E_{\alpha, \nu}(t, w)+c \nu\|w(t)\|_{\alpha-1}^{2},
$$

and

$$
\|w(t)\|_{\alpha-1} \leq\|w(0)\|_{\alpha-1}+\int_{0}^{t}\left\|w^{\prime}(t)\right\|_{\alpha-1} d s
$$

Then

$$
\left(\tilde{E}_{\alpha, \nu}(t, w)\right)^{1 / 2} \leq\left(E_{\alpha, \nu}(t, w)\right)^{1 / 2}+(c \nu)^{1 / 2}\left(\|w(0)\|_{\alpha-1}+\int_{0}^{t}\left(E_{\alpha, \nu}(s, w)\right)^{1 / 2} d s\right) .
$$

Let $u$ and $v$ be as in the statement of Theorem 5.1 and let us put $w:=v-u$ in (47). Let us fix a number $\varepsilon>0$, and let us set

$$
\varepsilon^{\prime}:=\frac{\varepsilon}{\left(2+(c \nu)^{1 / 2} T\right)^{2}} .
$$

Now by Theorem 5.1 there exists a positive number $\delta\left(\leq \varepsilon^{\prime}\right)$ such that

$$
E_{\alpha, \nu}(0, v-u)<\delta \Longrightarrow E_{\alpha, \nu}(t, v-u)<\varepsilon^{\prime} \quad(0 \leq t \leq T) .
$$

It is easy to check, by the estimate (47) and by the equality (46), that the number $\delta$ is as required. Q.E.D.

As a first consequence of the above theorem we obtain the uniqueness and the existence of the solution to problem (10)-(11).

Corollary 5.1 (uniqueness). Let us assume that the operator A satisfies condition (8), and that $m$ satisfies (12) and (23).

Let $T>0$ be given, and let $u$ and $v$ in $C^{0}\left([0, T] ; \mathcal{E}_{3 / 2}\right)$ solve Eq. (10), with the same initial data $\left(u_{0}, u_{1}\right)$. Then $u$ coincides with $v$. 
Proof. Set

$$
\bar{T}:=\max \{t \in[0, T]: u \equiv v \text { on }[0, t]\} .
$$

We claim that $\bar{T}=T$. If, for a contradiction, $\bar{T}<T$, we may restart the Cauchy problem at time $\bar{T}$ with initial data $\left(u(\bar{T}), u^{\prime}(\bar{T})\right)=\left(v(\bar{T}), v^{\prime}(\bar{T})\right)$. Then we apply Theorem 5.1 for $\alpha_{0}=3 / 2, \alpha=1$ and $W=\left\{\left(u(\bar{T}), u^{\prime}(\bar{T})\right)\right\}$. We get, for $t$ in some right neighborhood of $\bar{T}$,

$$
\left|(u-v)^{\prime}(t)\right|^{2} \leq E_{1, \nu}(t, u-v)=0,
$$

hence $u(t)=v(t)$, which contradicts the assumption $\bar{T}<T$. Q.E.D.

We remark that Theorem 5.1 provides a mere continuity result of the resolvent map

$$
\left(u(0), u^{\prime}(0)\right) \in \mathcal{E}_{\alpha} \longmapsto u \in C^{0}\left([0, T] ; \mathcal{E}_{\alpha}\right) .
$$

In the following theorem we obtain, under a suitable condition (which will be discussed in the Appendix) on the set $W$, the uniform continuity of the map in (48).

Theorem 5.3 (uniformly continuous dependence). Let us assume that the operator A satisfies condition (8), and that

(i) $m$ satisfies (12) and (23);

(ii) $W$ is a bounded set of $\mathcal{E}_{3 / 2}$.

Let the quantity $T^{*}$ be defined as in (22) and let $\alpha_{0}$ and $T$ be real numbers such that (24) and (25) holds true.

Moreover assume that for each $n \in \mathbb{N}$

$$
h_{n, \alpha}:=\nu\left|Q_{n} A_{c}^{(\alpha-1) / 2} A^{1 / 2} u_{0}\right|^{2}+\left|Q_{n} A_{c}^{(\alpha-1) / 2} u_{1}\right|^{2} \leq \sigma(n)
$$

with

$$
\lim _{n \rightarrow \infty} \sigma(n)=0
$$

Then, the number $\delta$ in the thesis of Theorem 5.1 (resp.: in the thesis of Theorem 5.2) may be choosen in such a way to depend only upon $\varepsilon, \nu, \Lambda, \sigma(\cdot), \alpha, T / T^{*}$.

Proof. It suffices to substitute in the estimate (44) of the proof of Theorem 5.1 the quantity $\sigma(n)$ for $h_{n}$. Q.E.D.

An immediate consequence of Theorem 5.3 is the local existence of the solution of the Cauchy problem (10)-(11). Given any $\left(u_{0}, u_{1}\right) \in \mathcal{E}_{3 / 2}$, let us set

$$
\mathcal{H}\left(u_{0}, u_{1}\right):=M\left(\left\langle A u_{0}, u_{0}\right\rangle\right)+\left|u_{1}\right|^{2} .
$$

Corollary 5.2 (existence). Let us assume that the operator A satisfies condition (8), and that $m$ satisfies (12) and (23).

Let $\left(u_{0}, u_{1}\right)$ be in $\mathcal{E}_{\alpha_{0}}$ with $\alpha_{0} \geq 3 / 2$.

Set

$$
T^{*}\left(u_{0}, u_{1}\right):=\frac{\nu^{3 / 2}}{L\left(m\left(\left\langle A u_{0}, u_{0}\right\rangle\right)\left\|A^{1 / 2} u_{0}\right\|_{1 / 2}^{2}+\left\|u_{1}\right\|_{1 / 2}^{2}\right)} .
$$

Then there exists a unique solution $u$ in $C^{0}\left(\left[0, T^{*}\left[; \mathcal{E}_{\alpha_{0}}\right)\right.\right.$ of the Cauchy problem (10)- (11).

Moreover the whole sequence of Ritz-Galerkin approximations converges to the solution $u$ in the norm of the space $C^{0}\left([0, T] ; \mathcal{E}_{\alpha_{0}}\right)$ for every $T<T^{*}\left(u_{0}, u_{1}\right)$. 
Proof. Let $P_{n}$ be the orthogonal projection given by (39), and let us set

$$
W:=\left\{\left(P_{n} u_{0}, P_{n} u_{1}\right): n \in \mathbb{N}\right\},
$$

For each $n \in \mathbb{N}$ we consider the Ritz-Galerkin approximating solution $u_{n}$ corresponding to the initial data $\left(P_{n} u_{0}, P_{n} u_{1}\right)$, i.e. the solution of the problem

$$
\left\{\begin{array}{l}
u_{n}^{\prime \prime}+m\left(\left\langle A u_{n}, u_{n}\right\rangle\right) A u_{n}=0 \quad(t>0) \\
u_{n}(0)=P_{n} u_{0} \\
u_{n}^{\prime}(0)=P_{n} u_{1}
\end{array}\right.
$$

Note that $u_{n}$ exists (and is unique) for all $t \geq 0$, by the classical CauchyLipschitz theorem and by the conservation of the Hamiltonian $\mathcal{H}\left(t, u_{n}\right)$, (see Proposition 6.1 below), which implies that $\left|u_{n}^{\prime}(t)\right| \leq$ Const.

Let $T$ be any number such that

$$
0<T<T^{*}\left(u_{0}, u_{1}\right) .
$$

Let us observe that $T^{*}(W)=T^{*}\left(u_{0}, u_{1}\right)$, so we have $T<T^{*}(W)$.

We claim that $\left(u_{n}\right)$ is a Cauchy sequence in $C^{0}\left([0, T] ; \mathcal{E}_{\alpha_{0}}\right)$. Since the set $W$ satisfies the hypoteses of Theorem 5.3, for every $\varepsilon>0$ there exists a $\delta>0$ which satisfies the thesis of Theorem 5.3. Then we choose $\bar{n}$ large enough to have

$$
\tilde{E}_{\alpha_{0}, \nu}\left(0, u_{n}-u_{m}\right)<\delta \quad(n \geq \bar{n}, m \geq \bar{n}) .
$$

By Theorem 5.3 we have therefore

$$
\tilde{E}_{\alpha_{0}, \nu}\left(t, u_{n}-u_{m}\right)<\varepsilon \quad \text { for } 0 \leq t \leq T .
$$

This is exactly our claim. Therefore $\left(u_{n}\right)$ converges to a function $u \in$ $C^{0}\left([0, T] ; \mathcal{E}_{\alpha}\right)$ for each $\alpha \leq \alpha_{0}$, in particular for $\alpha=1$. By the continuity of the function $m$, we have

$$
m\left(\left\langle A u_{n}(\cdot), u_{n}(\cdot)\right\rangle\right) \longrightarrow m(\langle A u(\cdot), u(\cdot)\rangle) \quad \text { uniformly on }[0, T] .
$$

To achieve the existence it suffices to pass to the limit in Eq.(10). The uniqueness was already established in Corollary 5.1. Q.E.D.

\section{Extensions of the Results of SeCtion 5}

In this section we will prove that the simplifying assumptions made in section 4 may be dispensed with.

First we prove that, due to the conservative character of the Kirchhoff equation, it is enough to require (12) and (23) in a local sense.

To this aim we recall the Hamiltonian (5), which in abstract form reads as

$$
\mathcal{H}(t, u):=M(\langle A u(t), u(t)\rangle)+\left|u^{\prime}(t)\right|^{2} \quad(t \geq 0) .
$$

We have

Proposition 6.1 ([Be]). Let $u \in C^{0}\left([0, T] ; \mathcal{E}_{3 / 2}\right)$ be a solution to the Cauchy problem (10)- (11). Then the Hamiltonian $\mathcal{H}(t, u)$ is constant with respect to $t$.

For any subset $W \subseteq \mathcal{E}_{3 / 2}$, let us introduce the following (possibly infinite) quantities. First we set

$$
\mathcal{H}:=\mathcal{H}(W):=\sup _{\left(u_{0}, u_{1}\right) \in W} M\left(\left\langle A u_{0}, u_{0}\right\rangle\right)+\left|u_{1}\right|^{2} .
$$


Then we introduce $\quad \nu:=\nu(W), \quad \Lambda:=\Lambda(W)$ and $L:=L(W)$ as the best constants such that

$$
\begin{gathered}
M(r) \leq \mathcal{H} \Longrightarrow \nu \leq m(r) \leq \Lambda, \\
{[M(r) \leq \mathcal{H}, M(s) \leq \mathcal{H}] \Longrightarrow|m(r)-m(s)| \leq L|r-s| .}
\end{gathered}
$$

Finally let $E(W)$ and $T^{*}(W)$ be defined as in (21), (22).

Proposition 6.2. Let us assume that the operator A satisfies condition (8), and that $1.1)$

(i) $m:[0,+\infty[\longrightarrow] 0,+\infty[$ is a locally Lipschitz function coercive at $\infty$ (Def.

(ii) $W$ is a bounded subset of $\mathcal{E}_{3 / 2}$.

Let the quantities $\mathcal{H}, E, \nu, \Lambda, L$ and $T^{*}$ be defined as above. Then

$$
\begin{gathered}
\mathcal{H}+E+\Lambda+L<\infty \\
0<\nu \\
0<T^{*} \leq \infty
\end{gathered}
$$

Moreover, if we restrict ourselves to solutions in $C^{0}\left([0, T] ; \mathcal{E}_{3 / 2}\right)$, for initial data in $W$ the Cauchy problem (10)-(11) is equivalent to an analogous problem for a modified function $\tilde{m}$ satisfying (12) and

$$
\tilde{m}(s) \leq \Lambda \quad(s \geq 0) .
$$

Proof. Assumption (ii) means that the quantity

$$
\left\|u_{0}\right\|_{3 / 2}^{2}+\left\|u_{1}\right\|_{1 / 2}^{2}, \quad\left(u_{0}, u_{1}\right) \in W
$$

is bounded. The same holds true for the quantity $\left\langle A u_{0}, u_{0}\right\rangle\left(\left(u_{0}, u_{1}\right) \in W\right)$, since, thanks to (8) and the spectral decomposition of the operators $A^{1 / 2}$ and $A_{c}^{1 / 2}$,

$$
\left\langle A u_{0}, u_{0}\right\rangle \leq K\left\|u_{0}\right\|_{3 / 2}^{2}, \quad \text { where } K:=\frac{\|i\|_{V, H}}{\eta(A)^{1 / 2}} .
$$

Therefore the quantities $\mathcal{H}$ and $E$ are finite. Then by assumption (i) it follows that the quantities $\Lambda, L$ and $T^{*}$ are finite, and that $\nu>0$.

Let $u \in C^{0}\left([0, T] ; \mathcal{E}_{3 / 2}\right)$ be any solution of the problem (10)-(11) with initial data in $W$. By Proposition 6.1,

$$
\mathcal{H}(t, u)=\mathcal{H}(0, u)=: \mathcal{H},
$$

so we have

$$
M(\langle A u(t), u(t)\rangle) \leq \mathcal{H} \quad(0 \leq t \leq T) .
$$

Again by (i) (cf. (4)) there exists a number $\tilde{r}$ such that

$$
M(r)>\mathcal{H} \quad \text { for } r>\tilde{r} .
$$

From (52) and (53) it follows that

$$
\langle A u(t), u(t)\rangle \leq \tilde{r} \quad(0 \leq t \leq T) .
$$


If we truncate the function $m$ in the following manner:

$$
\tilde{m}:= \begin{cases}m(r) & \text { if } \quad 0 \leq r<\tilde{r} \\ m(\tilde{r}) & \text { if } \quad r \geq \tilde{r}\end{cases}
$$

then $u$ satisfies also the equation

$$
u^{\prime \prime}+\tilde{m}(\langle A u(t), u(t)\rangle) A u=0 \quad(0<t<T) .
$$

If moreover $\tilde{r}$ is choosen as the smallest number such that (53) holds true (i.e. $\tilde{r}=M^{-1}(\mathcal{H})$ ), we have

$$
\begin{aligned}
\inf _{[0,+\infty[} \tilde{m} & =\inf _{[0, \tilde{r}]} m=: \nu, \\
\sup _{[0,+\infty[} \tilde{m} & =\sup _{[0, \tilde{r}]} m=: \Lambda, \\
\sup _{\substack{r, s \in[0, \tilde{r}] \\
r \neq s}} \frac{|\tilde{m}(r)-\tilde{m}(s)|}{|r-s|} & =\sup _{\substack{r, s \in[0, \tilde{r}] \\
r \neq s}} \frac{|m(r)-m(s)|}{|r-s|}=: L .
\end{aligned}
$$

The converse statement may be proven in an analogous way. Q.E.D.

Thanks to Proposition 6.2, it follows that in the statements of Theorems 5.1, 5.2, 5.3 and Corollaries 5.1, 5.2, one might assume $m$ locally Lipschitz and coercive at $\infty$.

Then we pass to consider the case when a source term $f$ is present. When we are dealing with Eq.(9), there are a few complications in the statement of the theorems. More explicitly, let $W$ be a bounded set of $\mathcal{E}_{3 / 2}$ and let $F$ be a subset of $L^{1}\left(\left[0, T^{*}\right] ; V_{\alpha_{0}-1}\right), F$ bounded in $L^{1}\left(\left[0, T^{*}\right] ; V_{1 / 2}\right)$, where $T^{*}=T^{*}(W)$ is given by (22). We assume that $f$ varies in $F$. In this case the Hamiltonian $\mathcal{H}(t)$ (see (5) is no longer constant anyway, we have the estimate

$$
\mathcal{H}^{\prime}(t)=2 \operatorname{Re}\left(u^{\prime}, f\right) \leq 2\left|u^{\prime}\right||f| \leq 2 \mathcal{H}(t)^{1 / 2}|f| .
$$

Therefore

$$
\mathcal{H}(t) \leq\left(\mathcal{H}(0)^{1 / 2}+\int_{0}^{t}|f(s)| d s\right)^{2} .
$$

The presence of the source term forces us to modify the constants $\nu, \Lambda, L$ and the critical time $T^{*}$. First of all, we set

$$
\tilde{\mathcal{H}}:=\tilde{\mathcal{H}}(W, F):=\sup _{g \in F}\left(\mathcal{H}(W)^{1 / 2}+\int_{0}^{T^{*}}|g(s)| d s\right)^{2},
$$

then we define $\tilde{\nu}, \tilde{\Lambda}, \tilde{L}$ as in (50) and (51) with $\tilde{\mathcal{H}}$ in place of $\mathcal{H}(W)$. Finally, the critical time $T^{* *}:=T^{* *}(W, F)$ is implicitly defined by

$$
\frac{\tilde{\nu}^{3 / 2}}{\tilde{L}}=T^{* *}\left(E^{1 / 2}+\sup _{g \in F} \int_{0}^{T^{* *}}\|g\|_{1 / 2} d s\right)^{2} .
$$

Now we are in a position to state the inhomogeneous version of Theorem 5.1, which reads as follows. For every $\varepsilon>0$, there exists a number $\delta$ which depends only upon the quantities $\varepsilon, \tilde{\nu}, \tilde{\Lambda}, u_{0}, u_{1}, \alpha, T / T^{* *}$, such that

$E_{\alpha, \nu}(0, v-u)+\int_{0}^{T}\|(g-f)(s)\|_{\alpha-1} d s<\delta \Longrightarrow E_{\alpha, \nu}(t, v-u)<\varepsilon \quad(0 \leq t \leq T)$,

for every $v$ in $C^{0}\left([0, T] ; \mathcal{E}_{\alpha_{0}}\right)$ which solves the Cauchy problem (9)-(11) with initial data in $W$ and source term $g$ in $F$. 
In a similar way one might modify the statements of Theorem 5.2, Corollary 5.1, Theorem 5.3, Corollary 5.2.

\section{Study of the continuity modulus of the Resolvent map (3), Which IN GENERAL IS NOT HÖLDER CONTINUOUS}

Theorem 5.1 guarantees the continuity of the resolvent map (48), but by no means gives informations about its continuity modulus. Actually, the continuity of the resolvent map (48) for the Kirchhoff equation cannot in general be improved to Hölder continuity. To show this we exhibit a counterexample, which is modelled on a previous one of Kato [K1] for the so-called Burgers' equation $u_{t}+u u_{x}=0$.

Let us set $\Omega \equiv \mathbb{R}$ (we might as well consider a bounded interval of $\mathbb{R}$ ) and $m(r):=1+r$. If we are looking for solutions of Eq.(1) (with $f \equiv 0$ ) of the form $u_{0}(x-c t)$, for some positive constant $c$, we find the conditions

$$
\begin{gathered}
c=c\left(u_{0}\right):=\left(1+\int_{-\infty}^{+\infty}\left|\frac{d u_{0}}{d x}\right|^{2} d x\right)^{1 / 2}, \\
u(x, 0)=u_{0}(x), \quad u_{t}(x, 0)=-c \frac{d u_{0}}{d x}(x) \quad(x \in \mathbb{R}) .
\end{gathered}
$$

Given a function $u_{0}$, we call $-c\left(u_{0}\right) d u_{0} / d x$ the compatible velocity for $u_{0}$.

Now let us define

$$
f(x):= \begin{cases}\frac{x^{3 / 2}}{\log x} \quad \text { if } x>0, \\ 0 \quad \text { if } x \leq 0,\end{cases}
$$

let us consider a function $\phi \in C_{0}^{\infty}(\mathbb{R})$ such that $\phi \equiv 1$ for $|x| \leq 1 / 2$, and finally let us consider for $\delta \in[-1,1]$, the family of functions $u_{0}^{\delta} \in H^{2}(\mathbb{R})$ defined as

$$
u_{0}^{\delta}(x):=(\delta+f(x)) \phi(x) \quad(x \in \mathbb{R}) .
$$

Note that

$$
\left\|u_{0}^{\delta}-u_{0}^{0}\right\|_{H^{2}}=|\delta|\|\phi\|_{H^{2}} .
$$

If we choose as initial data for the Kirchhoff equation the family $u_{0}^{\delta}$ with the relative compatible velocities, we get the following family of solutions:

$$
u^{\delta}(x, t)=\left(\delta+f\left(x-c_{\delta} t\right)\right) \phi\left(x-c_{\delta} t\right),
$$

where $c_{\delta}:=c\left(u_{0}^{\delta}\right)($ see $(54))$.

A simple computation gives for two suitable constants $k_{1}, k_{2}>0$

$$
k_{1}|\delta| \geq\left|c_{\delta}-c_{0}\right| \geq k_{2}|\delta|^{2} \quad \text { as } \delta \rightarrow 0 .
$$

Let us evaluate the $H^{2}$-norm of the difference of the solutions corresponding to $u_{0}^{\delta}$ and $u_{0}^{0}$. Let us fix $T>0$. If $\delta$ is so small that $\left|c_{\delta}-c_{0}\right| T<1$, we have

$$
\begin{aligned}
\sup _{t \in[0, T]}\left\|u^{\delta}(t)-u^{0}(t)\right\|_{H^{2}}^{2} & \geq \int_{0}^{\left|c_{\delta}-c_{0}\right| T}\left|\frac{d^{2} f}{d x^{2}}(x)\right|^{2} d x \\
& =\int_{0}^{\left|c_{\delta}-c_{0}\right| T} x^{-1}\left(\frac{3}{4 \log x}+\frac{2}{\log ^{2} x}-\frac{2}{\log ^{3} x}\right)^{2} d x .
\end{aligned}
$$


Then for $\delta$ small enough, by (57) the right-hand side behaves like

$$
\begin{aligned}
\left(\frac{3}{4}\right)^{2} \int_{0}^{\left|c_{\delta}-c_{0}\right| T} \frac{d x}{x \log ^{2} x} & =\left(\frac{3}{4}\right)^{2} \frac{1}{-\log \left(\left|c_{\delta}-c_{0}\right| T\right)} \\
& \geq\left(\frac{3}{4}\right)^{2} \frac{1}{-\log \left(k_{2}|\delta|^{2} T\right)} .
\end{aligned}
$$

By (56) one cannot therefore expect Hölder continuity of the map (48) for any positive exponent.

To conclude, let us investigate which conditions guarantee the Hölder continuity of the map (48). For example, via an interpolation estimate, it is not difficult to prove that if $W \subseteq \mathcal{E}_{\alpha+\beta}$ for some $\left.\left.\beta \in\right] 0,1\right]$, then the map (48) is Hölder continuous with exponent $\beta$. In the next theorem we prove the stronger result that it suffices to take the fixed initial datum $\left(u_{0}, u_{1}\right)$ in $\mathcal{E}_{\alpha+\beta}$ to have Hölder continuity of the map (48) at $\left(u_{0}, u_{1}\right)$.

Theorem 7.1. Let us assume that the operator A satisfies condition (8), and that

(i) $m:[0,+\infty[\longrightarrow] 0,+\infty[$ is a locally Lipschitz function coercive at $\infty$;

(ii) $W$ is a bounded set of $\mathcal{E}_{3 / 2}$.

Let the quantities $E, T^{*} \mathcal{H}, \nu, \Lambda$ and $L$ be defined as in (21), (22), (49)-51). Let $\alpha$ and $T$ be real numbers such that

$$
\begin{gathered}
\alpha \geq 3 / 2, \\
0<T<T^{*} .
\end{gathered}
$$

Assume that the initial datum $\left(u_{0}, u_{1}\right)$ belongs to $\mathcal{E}_{\alpha+\beta}$ with $0<\beta \leq 1$.

Then

$$
E_{\alpha, \nu}(t, u-v) \leq C\left\{\left(E_{\alpha, \nu}(0, u-v)\right)^{\beta}+E_{\alpha, \nu}(0, u-v)\right\} \quad(0 \leq t \leq T),
$$

where the constant $C$ depends upon the quantities $\nu, \Lambda, T / T^{*}, E_{\alpha+\beta, \nu}(0, u), E$ and $\alpha$.

The exponent $\beta$ in (58) cannot be improved.

Remark 7.1. In the case when $\beta=1$, we have Lipschitz continuity of the map (48) (note that this agrees with the last statement of Theorem 5.1 for the case $\alpha=1 / 2$, since the initial data belong at least to $\mathcal{E}_{3 / 2}$ ).

Proof. Let $n \in\left[0,+\infty\left[\right.\right.$ and let $h_{n, \alpha}\left(u_{0}, u_{1}\right)$ be defined as in (42). We have for $n^{2}+c>0$

$$
h_{n, \alpha}\left(u_{0}, u_{1}\right) \leq\left(n^{2}+c\right)^{-\beta} E_{\alpha+\beta, \nu}(0, u) .
$$

Let us put $\alpha+\beta-1$ in place of $\alpha$ in the estimate (37) of the proof of Theorem 5.1. We get, by (29) and (36), for $0 \leq t \leq T$

$$
E_{\alpha+\beta-1, m(v)}\left(t, w_{2}\right)=: y_{\alpha+\beta-1}^{2}(t) \leq \frac{E_{\alpha+\beta, m_{(u)}}(0, u) E_{1 / 2, m_{(v)}}(0, v-u)}{E\left(1-T / T^{*}\right)^{5}} .
$$


By following the lines of the estimate (41) with $\alpha+\beta-1$ in place of $1 / 2$, we have then

$$
\begin{aligned}
E_{\alpha, \nu}\left(t, P_{n} w_{2}\right) & \leq\left(n^{2}+c\right)^{1-\beta} E_{\alpha+\beta-1, m_{(v)}}\left(t, w_{2}\right) \\
& \leq \frac{\Lambda}{\nu}\left(n^{2}+c\right)^{1-\beta} \frac{E_{\alpha+\beta, m_{(u)}}(0, u) E_{1 / 2, \nu}(0, v-u)}{E\left(1-t / T^{*}\right)^{5}} .
\end{aligned}
$$

Moreover, thanks to (43) and (59) we have for $n^{2}+c>0$

$$
E_{\alpha, \nu}\left(t, Q_{n} w_{2}\right) \leq \frac{4 \Lambda}{\nu}\left(n^{2}+c\right)^{-\beta} \frac{E_{\alpha+\beta, \nu}(0, u)}{1-t / T^{*}} .
$$

Therefore, by (27), (28), (40), (60), (61) and (45) for $\beta=\alpha$, we get for $0 \leq t \leq T$

$$
\begin{aligned}
E_{\alpha, \nu}(t, v-u) \leq & \frac{2 \Lambda}{\nu\left(1-T / T^{*}\right)}\left\{\left(n^{2}+c\right)^{1-\beta} \frac{E_{\alpha+\beta, m_{(u)}}(0, u) E_{1 / 2, \nu}(0, v-u)}{E\left(1-T / T^{*}\right)^{4}}\right. \\
& \left.+4\left(n^{2}+c\right)^{-\beta} E_{\alpha+\beta, \nu}(0, u)+E_{\alpha, \nu}(0, v-u)\right\} \\
\leq & C\left\{\left(n^{2}+c\right)^{1-\beta} E_{\alpha, \nu}(0, v-u)+\left(n^{2}+c\right)^{-\beta}+E_{\alpha, \nu}(0, v-u)\right\},
\end{aligned}
$$

where $C$ is a constant depending only upon $\Lambda / \nu, T / T^{*}, E_{\alpha+\beta, \nu}(0, u), E$ and $\alpha$.

If we minimize with respect to the (real) variable $n$, and then maximize with respect to $\beta \in] 0,1]$, we get

$$
\begin{aligned}
E_{\alpha, \nu}(t, u-v) & \leq C\left\{\frac{\left(E_{\alpha, \nu}(0, u-v)\right)^{\beta}}{\beta^{\beta}(1-\beta)^{1-\beta}}+E_{\alpha, \nu}(0, u-v)\right\} \\
& \leq 2 C\left\{\left(E_{\alpha, \nu}(0, u-v)\right)^{\beta}+E_{\alpha, \nu}(0, u-v)\right\}
\end{aligned}
$$

This proves estimate (58).

To show that the exponent $\beta$ is optimal we will exhibit, for each $\beta$, an initial datum belonging to $\mathcal{E}_{\alpha+\beta}$, for which condition (58) fails to hold true for any $\beta^{\prime}>\beta$.

We will do it only for $\alpha=2$, by adapting the previous counterexample. Let us define for $\beta>0$

$$
f_{\beta}(x):=\left\{\begin{array}{l}
\frac{x^{3 / 2+\beta}}{\log x} \text { if } x>0, \\
0 \text { if } x \leq 0,
\end{array}\right.
$$

and let us consider the following family of functions $u_{0}^{\delta, \beta}, \delta \in[-1,1]$, given by

$$
u_{0}^{\delta, \beta}(x):=\left(\delta+f_{\beta}(x)\right) \phi(x) .
$$

In order to improve (57) to

$$
k_{1}|\delta| \geq\left|c_{\delta}-c_{0}\right| \geq k_{3}|\delta| \quad \text { as } \delta \rightarrow 0 \quad\left(k_{3}>0\right)
$$

we assume now that

$$
\int_{0}^{+\infty} \frac{d \phi}{d x} \frac{d(\phi f)}{d x} d x \neq 0
$$



(3))

Since $u_{0}^{\delta, \beta} \in H^{2+\beta}(\mathbb{R})$, the pair $\left(u_{0}^{\delta, \beta}, c\left(u_{0}^{\delta, \beta}\right) d u_{0}^{\delta, \beta} / d x\right)$ belongs to $\mathcal{E}_{2+\beta}$ (see

On the other hand, by arguing as in the previous counterexample we get, for $\delta \rightarrow 0^{+}$

$$
\frac{\left(k_{2}|\delta| T\right)^{\beta}}{-\log ^{2}\left(k_{2}|\delta| T\right)}=\mathrm{O}\left(\sup _{t \in[0, T]}\left\|u^{\delta, \beta}(t)-u^{0, \beta}(t)\right\|_{H^{2}}\right) .
$$

The above inequality proves that (58) may not hold for any $\beta^{\prime}>\beta$. Q.E.D.

\section{Appendix A.}

Let $H$ be any real or complex Hilbert space with norm $|\cdot|$ and inner product $(\cdot, \cdot)$. Let $B: D(B) \subseteq H \rightarrow H$ be any self-adjoint operator. We denote by $E(\lambda)$ the spectral decomposition relative to $B$; we refer to $[\mathrm{Ru}]$ for the definition and the properties of $E(\lambda)$. For any $x \in H$, let $E_{x, x}$ be the positive measure given by

$$
E_{x, x}(\omega)=(E(\omega) x, x) \quad \text { for any Borel subset } \omega \text { of } \mathbb{R} .
$$

For every $s \geq 0$, let us define the following orthogonal projection in $H$

$$
Q_{s}:=\int \chi_{] s,+\infty} d E(\lambda)
$$

where $\chi_{G}$ denotes the characteristic function of a set $G \subseteq \mathbb{R}$.

Theorem A.1. For any bounded subset $W$ of $H$, the following conditions are equivalent:

$$
\lim _{s \rightarrow+\infty}\left|Q_{s} x\right|=0 \quad \text { uniformly in } \quad x \in W
$$

There exists a measurable real function $f$ such that

$$
\lim _{r \rightarrow+\infty} f(r)=+\infty, \quad W \text { is a bounded subset of } D(f(B)) .
$$

Proof. (A2) $\Longrightarrow(\mathrm{A} 1)$. We have for every $x \in W$, for $s$ large enough

$$
\begin{aligned}
\left|Q_{s} x\right|^{2} & =\int \chi_{] s,+\infty[} d E_{x, x} \\
& \leq \frac{1}{\inf _{s,+\infty[} f^{2}} \int \chi_{] s,+\infty[} f^{2} d E_{x, x} \\
& \leq \frac{1}{\inf _{s,+\infty[} f^{2}}|f(B) x|^{2} .
\end{aligned}
$$

Now (A2) implies that

$$
|f(B) x|^{2} \leq \text { Const. } \quad(x \in W),
$$

and

$$
\lim _{s \rightarrow+\infty} \frac{1}{\inf _{s,+\infty[} f^{2}}=0 .
$$

(A1) $\Longrightarrow(\mathrm{A} 2)$. Let $k_{0}$ be the first natural number such that

$$
\sup _{x \in W}\left|Q_{k_{0}} x\right| \leq 1
$$


and by induction for $i=1,2, \ldots$ let us define $k_{i}$ as the first natural number such that

$$
k_{i}>k_{i-1} \quad \text { and } \quad \sup _{x \in W}\left|Q_{k_{i}} x\right| \leq 2^{-i} .
$$

We construct the function $f$ in the following way:

$$
f(r):= \begin{cases}i & \text { if } \left.r \in] k_{i}, k_{i+1}\right] \\ 0 & \text { if } r \leq k_{0}\end{cases}
$$

For every $x \in W$, we have

$$
|f(B) x|^{2}=\int f^{2} d E_{x, x}=\sum_{i=0}^{+\infty} \int \chi_{] k_{i}, k_{i+1}\right]} f^{2} d E_{x, x} \leq \sum_{i=0}^{+\infty} i^{2} 2^{-i}<+\infty .
$$

Since $\lim _{r \rightarrow+\infty} f(r)=+\infty$, the proof is complete . Q.E.D.

The next theorem states two conditions under which (A1) (equivalently: (A2)) holds true.

Theorem A.2. For any bounded subset $W$ of $H$, let us consider the following conditions:

$$
\text { There exists a nonnegative Borel measure } \mu \text { on } \mathbb{R} \text {, such that }
$$

$$
\mu(\mathbb{R})<+\infty \quad \text { and }
$$

on some positive half-line: $E_{x, x} \leq \mu$ for each $x \in W$.

Then each of the above conditions are sufficient to imply (A1), but none is necessary (but if $B^{-1}$ exists and is a compact operator, then (A3) is necessary).

Proof. (A3) $\Longrightarrow(\mathrm{A} 1)$. Since for every $x \in H$

$$
\left|Q_{s} x\right|^{2}=\int \chi_{] s,+\infty[} d E_{x, x}=E_{x, x}(] s,+\infty[) \rightarrow 0 \quad \text { as } s \rightarrow+\infty
$$

it follows that $\left|Q_{s} x\right| \rightarrow 0$ uniformly on any relatively compact subset of $H$.

$(\mathrm{A} 4) \Longrightarrow(\mathrm{A} 1)$. We have for $s$ large enough

$$
\left|Q_{s} x\right|^{2}=E_{x, x}(] s,+\infty[) \leq \mu(] s,+\infty[) \quad(x \in W) .
$$

Since $\mu$ is finite we have $\lim _{s \rightarrow+\infty} \mu(] s,+\infty[)=0$, so that (A1) holds true.

We remark that between the conditions (A3) and (A4) there is in general no implication, as the following examples show. A fortiori none of these conditions is necessary to have (A1).

$(\mathrm{A} 3) \Longrightarrow(\mathrm{A} 4)$. Let $B$ be any unbounded positive operator. Then there exists a sequence $\left(\omega_{k}\right)$ of subsets of $\mathbb{R}$ such that:

(i) the $\omega_{k}$ are mutually disjoint;

(ii) $E\left(\omega_{k}\right) \neq 0$ for each $k \in \mathbb{N}$;

(iii) for each $s \in \mathbb{R}$ there exists $\bar{k}(s)$ such that $\omega_{k} \subseteq[s,+\infty$ [ for each $k \geq \bar{k}(s)$ (e.g. $\omega_{k}=\left[\lambda_{k}, \lambda_{k+1}\left[\right.\right.$, where $\left(\lambda_{k}\right)$ is a suitable sequence of real numbers $\left.\nearrow+\infty\right)$. 
By (ii) there exists $x_{k} \in H, E\left(\omega_{k}\right) x_{k} \neq 0$. We set

$$
v_{k}:=k^{-1 / 2} \frac{E\left(\omega_{k}\right) x_{k}}{\left|E\left(\omega_{k}\right) x_{k}\right|} \quad(k \in \mathbb{N}) .
$$

We have $\left|v_{k}\right|=k^{-1 / 2}$, so that $W:=\left\{v_{k}: k \in \mathbb{N}\right\}$ is relatively compact in $H$. On the other hand, if $\mu$ is any nonnegative Borel measure such that $E_{v_{k}, v_{k}} \leq \mu$ $(k \in \mathbb{N})$ on some half-line $[s,+\infty[$, then by (iii)

$$
k^{-1}=\left|v_{k}\right|^{2}=E_{v_{k}, v_{k}}(\mathbb{R})=E_{v_{k}, v_{k}}\left(\omega_{k}\right) \leq \mu\left(\omega_{k}\right) \quad(k \geq \bar{k}(s)),
$$

hence by (i)

$$
\mu(\mathbb{R}) \geq \sum_{k \geq 0} \mu\left(\omega_{k}\right) \geq \sum_{k \geq \bar{k}} k^{-1}=+\infty .
$$

$(\mathrm{A} 4) \not(\mathrm{A} 3)$. Let $\operatorname{dim} H=\infty, B$ any bounded operator, $W:=\{x \in H$ : $|x|=1\}$ and $\mu \equiv 0$.

To finish, we prove that (A3) is a necessary condition to have (A1), if $B^{-1}$ is a compact operator. Indeed if (A1) holds true then

$$
W=P_{s} W+Q_{s} W
$$

Now $B P_{s}$ is a bounded operator, hence $P_{s}=B^{-1} B P_{s}$ is a compact operator. Therefore $P_{s} W$ is precompact. Since $Q_{s} W$ is arbitrarily small as $s \rightarrow+\infty$, it follows by a standard argument that $W$ is precompact. Q.E.D.

\section{ACKNOWLEDGMENTS}

We wish to thank L. A. MEDEIROS for pointing out references [Po2] and [Ma], and Y. YAMADA for [Yam].

\section{REFERENCES}

[Am] W. F. Ames, Nonlinear partial differential equations in engineering, ch. $3 \S 10$, Academic Press, New York, 1965 MR 35:1235

[Ar] A. Arosio, Averaged evolution equations. The Kirchhoff string and its treatment in scales of Banach spaces, expanded text of a lecture given in " $2^{\circ}$ workshop on functional-analytic methods in complex analysis" (Trieste, 1993), W. Tutschke ed., World Scientific, Singapore (to appear)

[AG] A. Arosio and S. Garavaldi, On the mildly degenerate Kirchhoff string, Math. Meth. Appl. Sci. 14 (1991), 177-195 MR 92c:35072

[AS] A. Arosio and S. Spagnolo, Global solutions of the Cauchy problem for a non-linear hyperbolic equation, in "Nonlinear Partial Differential Equations and their Applications". Collège de France Seminar, Vol. VI, 1-26, H. Brezis \& J.L. Lions eds., Research Notes Math. 109, Pitman, Boston, 1984 MR 86e:35091 [Zbl 598:35062]

[Be] S. Bernstein, Sur une classe d'équations fonctionelles aux dérivées partielles (Russian), Izv. Akad. Nauk SSSR Ser. Mat. 4 (1940), 17-26 MR 2:102

[BL] N. Bazley \& H. Lange, The original Schrödinger equation revisited, Appl. Anal. 21 (1986), 225-233 MR 87m:35062

[Ca] G. F. Carrier, On the nonlinear vibration problem of the elastic string, Quart. Appl. Math. 3 (1945), 157-165; A note on the vibrating string, Quart. Appl. Math. 7 (1949), 97-101 MR 10:458a

[DS1] P. D'Ancona \& S. Spagnolo, Global solvability for the degenerate Kirchhoff equation with real analytic data, Invent. Math. 108 (1992), 247-262; On an abstract weakly hyperbolic equation modelling the nonlinear vibrating string, in "Developments in partial differential equations and applications to mathematical physics" (Proc.: Ferrara, 1991), G. Buttazzo, G. P. Galdi \& L. Zanghirati eds., Plenum Press, 1993

[DS2] P. D'Ancona \& S. Spagnolo, A class of nonlinear hyperbolic problems with global solutions, Arch. Rat. Mech. Anal. 124 (1993), 201-219 
[Di1] R. W. Dickey, Infinite systems of nonlinear oscillations equations related to the string, Proc. Amer. Math. Soc. 23 (1969), 459-468 MR 40:458

[Di2] R. W. Dickey, The initial value problem for a nonlinear semi-infinite string, Proc. Roy. Soc. Edinburgh, A82 (1-2) (1978), 19-26 MR 80d:45005

[Eb] Y. Ebihara, On the existence of local smooth solutions for some degenerate quasilinear hyperbolic equations, An. Acad. Bras. Ciênc. 57 (1985), 145-152 MR 88c:35105

[EMM] Y. Ebihara, L. A. Medeiros \& M. Miranda, Local solution for a nonlinear degenerate hyperbolic equation, Nonlinear Anal. T.M.A. 10 (1986), 27-40 MR 86j:35264

[Fu] D. Fujiwara, Concrete characterization of the domains of fractional powers of some elliptic differential operators of the second order, Proc. Japan Acad. 43 (1967), 82-86 MR 35:7170

[Ga] S. Garavaldi, Su un modello integrodifferenziale non lineare della corda/membrana vibrante, Tesi di Laurea, Univ. Parma, December 1989

[GH] J. M. Greenberg \& S. C. Hu, The initial-value problem for a stretched string, Quart. Appl. Math. 38 (1980), 289-311 MR 82a:35021

[K1] T. Kato, The Cauchy problem for quasi-linear symmetric hyperbolic systems, (example 5.2) Arch. Rat. Mech. Anal. 58 (1975), 181-205 MR 52:11341

[K2] T. Kato, Quasi-linear equations of evolution, with applications to partial differential equations, in "Spectral Theory and Differential Equations" (Proc.: Dundee, 1974), 25-70, Lecture Notes Math. 448, Springer, 1975 MR 53:11252; Quasi-linear equations of evolution of hyperbolic type, in "Hyperbolicity" (C.I.M.E.: Cortona, 1976), 125-191 (Theorem 3.1, 3.2) Liguori, Napoli, 1977 [Zbl 34S:35052]

[K3] T. Kato, Abstract Differential Equations and Nonlinear Problems (Theorem 5.2) Lezioni Fermiane, Sc. Norm. Sup. Pisa, 1985 MR 88m:34058

[Ka] H. Kauderer, Nichlineare Mechanik, Part 2, B II, $\S 1.88$ b, Springer, Berlin, 1958 MR 26:3238

[Ki] G. Kirchhoff, Vorlesungen ober mathematische Physik: Mechanik, ch. $29 \S 7$, Teubner, Leipzig, 1876

[Li] J. L. Lions, On some questions in boundary value problems of mathematical physics, in "Contemporary developments in continuum mechanics and PDE's", G.M. de la Penha \& L.A. Medeiros eds., North-Holland, Amsterdam, 1978 MR 80a:73003 [Zbl 404:35002]

[LM] J. L. Lions and E. Magenes Problèmes aux limites non homogènes et applications Vol. I, ch. 1, 70-77, Dunod, Paris, 1968 MR 40:512

[Ma] M. P. Matos, Mathematical analysis of the nonlinear model for the vibrations of a string, Nonlinear Anal. T.M.A. 17, No.12 (1991), 1125-1137 MR 92k:35191

$[\mathrm{MM}]$ L. A. Medeiros \& M. Miranda, Solutions for the equations of nonlinear vibrations in Sobolev spaces of fractionary order, Computational Appl. Math. 6 (1987), 257-267 MR 90a:35150

[Me] G. P. Menzala, Une solution d'une équation nonlinéaire d'évolution, C. R. Acad. Sci. Paris 286 (1978), 273-275; On a classical solutions of quasilinear hyperbolic equation, Nonlinear Anal. T.M.A. 3 (1979), 613-627 MR 81e:35075

[Na] R. Narasimha, Nonlinear vibration of an elastic string, J. Sound Vibration 8 (1968), 134-146 [Zbl 164:267]

[NM] A. H. Nayfeh \& D. T. Mook, Nonlinear oscillations, $\S 7.4-\S 7.6$, Wiley-Interscience, New York, 1979 MR 80m:70002

[Ni] T. Nishida, Nonlinear vibrations of an elastic string II, unpublished manuscript (19711978)

[Nis] K. Nishihara, On a global solution of some quasilinear hyperbolic equation, Tokyo J. Math. 7 (1984), 437-459 MR 86g:35124

[Op] D. W. Oplinger, Frequency response of a nonlinear stretched string, J. Acoustic Soc. Amer. 32 (1960), 1529-1538 MR 22:11649

[Po1] S. I. Pokhozhaev, On a class of quasilinear hyperbolic equations, Mat. Sbornik 96 (138) (1) (1975), 152-166) (English transl.: Math. USSR Sbornik 25 (1975), 145-158) MR 51:6167 [Zbl 309:35051]

[Po2] S. I. Pokhozhaev, The Kirchhoff quasilinear hyperbolic equation, Differentsial'nye Uravneniya 21, No.1 (1985), 101-108 (English transl.: Differential Equations 21 (1985), 82-87) MR 86h:35056 
[Ri] P. H. Rivera Rodriguez, On a nonlinear hyperbolic equation, An. Acad. Brasil. Ciênc. 50 (1978), 133-135 MR 58:17399; On local strong solutions of a nonlinear partial differential equation, Appl. Anal. 10 (1980), 93-104 MR 81h:34070

[Ru] W. Rudin, Functional Analysis, ch. 13, Mc Graw-Hill, New York, 1973 MR 51:1315

[Ya] Y. Yamada, Some nonlinear degenerate wave equations, Nonlinear Anal. T.M.A. 10 (1987), 1155-1168 [Zbl 641:35044] MR 89a:35142

[Yam] T. Yamazaki, On local solutions of some quasilinear degenerate hyperbolic equations, Funkcial. Ekvac. 31 (1988), 439-457 MR 90g:35109

Dipartimento di Matematica, Università di Parma, Parma, Italy

E-mail address: panizzi@prmat.math.unipr.it 